\title{
Design and Collaborative Operation of Multimobile Inspection Robots in Smart Microgrids
}

\author{
Nankai Chen (iD) and Yaonan Wang (D) \\ College of Electrical and Information Engineering, Hunan University, Changsha 410082, China \\ Correspondence should be addressed to Nankai Chen; chennk@hnu.edu.cn
}

Received 19 November 2020; Revised 21 February 2021; Accepted 8 March 2021; Published 17 March 2021

Academic Editor: Xin Li

Copyright (c) 2021 Nankai Chen and Yaonan Wang. This is an open access article distributed under the Creative Commons Attribution License, which permits unrestricted use, distribution, and reproduction in any medium, provided the original work is properly cited.

\begin{abstract}
This paper investigates the substation inspection problems of multimobile robots for large power stations in smart microgrids. Most multirobot inspection robots generally face the challenge of path planning, while the current widely used biological excitation neural network (BENN) methods often have the defect of the neuronal active field near boundaries and obstacles. To end this, we propose an improved biological excitation neural network (IBENN) method for path planning based on a detailed architecture and ontology framework, through which the single-point inspection, multipoint inspection, and full-area inspection tasks of substations in smart microgrids can be well completed. Simulation results show that the designed IBENN-based multirobot collaboration inspection (MRCI) system can effectively shorten the path as well as the number of turns and then show better performance than most existing results when implementing various substation inspection tasks.
\end{abstract}

\section{Introduction}

Safe and stable supply of electricity is of great significance to ensure the sound and rapid development of the economy and society, whereas the grid and substation generally suffer from long-term exposure to the wild during the transmission and transformation process. It is necessary to detect and maintain transmission lines and substations regularly to prevent large-scale grid accidents, among which substation equipment maintenance is an important mean to ensure safe operation of smart microgrids, improve the economy of power grid operation, and provide good services [1-4].

Most of the current maintenance work still relies on manual operation, which has the disadvantage of dangerous working environment and high maintenance cost. Moreover, the factors of high intensity, low efficiency, and cannot work around the clock are also the main difficulties that manual inspection must face. In view of this, intelligent automation technology has gradually replaced manpower to become a new trend of inspection work due to the rapid technology development. By simulating operation behavior, robots have been used in the detection process of smart grids, which cannot only reduce work risks and human consumption but also provide accurate data detection and data collection. However, most inspection robots used in power stations are manually remote-controlled rather than autonomously operated and are used alone, lacking cooperation between multiple robots.

Inspection robots often carry out comprehensive and detailed inspection regularly for the equipment in the substation of smart microgrids to achieve all-weather, nodead, and efficient inspection work. Therefore, the multirobot intelligent inspection system should possess the functions of (i) autonomic path planning (planning a safe and optimal path traverses all task points with known information and information shared by other robots); (ii) environmental perception (sensing task points, obstacles, etc.); (iii) fault detection (it should be able to collect data through visible-light camera, infrared thermal imager, ultrasonic device, and other devices and transmit the acquired information to the background through the communication module for observation and analysis); (iv) remote operation 
(the staff can realize the continuous monitoring of the specific fault after the abnormal situation is found by the remote operation robot).

Inspection robots have been researched for more than thirty years. The substation inspection robot was first developed by Mitsubishi Corporation of Japan, Quek North Hydro Corporation of Canada, and University of São Paulo of Brazil [5-7]. A teleoperated robotic platform called LineScout Technology was introduced in [8], which has the capability to clear most obstacles that were found on the grid; however, the main functions and application modules were limited to the specified platform. Following this line, a method of detecting power grid lines by using the unmanned aerial vehicle (UAV) in the complex environment was presented in [9-11]. Furthermore, the inspection operations of flying robots and climbing robots for power stations have also been made preliminary attempts [12], although mobile robots were more widely used in the field of intelligent inspection of power stations of smart microgrids, no matter wheel-type or crawlertype robots. The above methods were based on single robots, which have become increasingly unable to meet the demand requirement of operation with the increase of the number of tasks and operation difficulty. Multirobot collaboration inspection (MRCI) has become an important research direction in robot research because of the natural coordination advantages; however, it is more complex and difficult than single robots in terms of scheduling and control, especially for the challenging path planning issues, which is the focus of this article. Some research achievements have been made in path planning of multiple robots. Yu and LaValle [13] investigated on optimal multirobot path planning over four minimization objectives by combining the ILP model algorithm with the heuristic algorithm. Their approach could achieve good levels of efficiency. Kelin and Pratihar [14] integrated the genetic algorithm with the $\mathrm{A}^{*}$ algorithm to design an approach used to plan the paths of multiple robots. Pradhan et al. [15] researched the problem of multiple robots moving towards individual goals within a common workspace, whereas the motion of every individual robot is deduced by a novel Particle Swarm Optimization-tuned Feed-Forward Neural Network. Luo et al. [16] proposed a neural dynamics approach for complete area coverage navigation by multiple robots and achieved high computational efficiency. Woosley and Dasgupta [17] proposed an abstraction that models the uncertainty in the paths and a Markov decision process-based algorithm that selects paths for the multirobots to find the task ordering that could reduce the overall distance. In the study by Rogers et al. [18], they also developed an approach, where the multiple robots were guided in their decision to select a location by a utility function that combines Gaussian processbased distributions for information entropy and communication signal strength. In a study performed by Imeson and Smith [19], a framework for solving multirobot motion planning problems with complex constraints had been introduced. They also proposed an algorithm that leverages recent advances in Satisfiability Modulo Theory to combine state-of-the-art SAT and TSP solvers. Ayari and Bouamama [20] proposed a new dynamic distributed particle swarm optimization algorithm for trajectory path planning of multiple robots in order to find the collision-free optimal path for each robot in the environment. More recently, some heuristic intelligent algorithms were adopted for path planning of the MRCI system, including the path conflict-based search algorithm [21] and the probability window-based multiobjective particle swarm optimization algorithm [22], to name just a few. Nevertheless, autonomous navigation is still one of the most challenging abilities for mobile robots.

Robot navigation consists of four parts: perception, positioning, decision-making, and execution, i.e., all robots must have the ability to sense the surrounding environment through sensors and determine its own position, then work out the motion strategy, and finally control the motor output to achieve the desired trajectory [23]. Several typical robot navigation methods include railway track, magnetic navigation, and pattern recognition. Since the railway track navigation method lacks of flexibility and needs hard work for the orbit construction [24], the magnetic navigation was introduced in $[25,26]$ considering the substation environment adaptability and cost, where the RFID tag information (detected by the RFID reader) is uploaded to the control system via the communication port to achieve the positioning of the inspection robot parking or turning movements. The pattern recognition-based navigation method employs noncontact sensors such as cameras and lidars to identify the road and, in turn, determines the robot's forehead posture, the current road, and the next location to be inspected. However, it requires a large amount of calculation and has high uncertainty [27].

As the substation environment is relatively fixed, the static map of the whole substation can be planned offline. In addition, since the substation studied in this paper is an outdoor environment, GPS navigation which has the advantages of high positioning accuracy, and a simple structure is adopted after comprehensive consideration of the mobile robot's path flexibility and comprehensive cost. Based on this, this paper mainly studies the multirobot autonomous navigation problem in power stations of smart microgrids, and multimobile robots are used to complete the collaborative inspection operation in the outdoor large-scale substation. In the following, the inspection tasks are divided into three categories: single-point inspection, multipoint inspection, and all-area inspection. We will design different MRCI algorithms to complete different inspection tasks efficiently.

The rest of the parts are organized as follows. Section 2 formulates the multirobot collaborative inspection problem, and the main IBENN-based navigation method is proposed in Section 3. Section 4 shows the implementation process and verifies the effectiveness of the established IBENN-based MRCI system in three different inspection tasks, and the paper is concluded in Section 5.

\section{Problem Formulation and Modeling}

We first establish the intelligent inspection robot system modeling in Subsection A and introduce three important inspection tasks and the commonly used BENN method, respectively, in Subsections B and C. 
2.1. Intelligent Inspection Robot System Modeling. The system is divided into two parts: remote control platform and robot body, as shown in Figure 1. The remote console is composed of a host PC and a database, which realizes the functions of information storage, status display, overall planning, and centralized control. The remote control platform controls the inspection robot through a $2.4 \mathrm{G}$ wireless communication module, and the inspection robot sends the videos and images of the inspection operation to the remote control platform through wireless Ethernet. The video capture card is connected to the on-board PC through the PCIE bus, which is used to collect the images of visible-light camera, infrared thermal imager, and the front camera of the mechanical arm. The motor driver is connected to the on-board PC via the USB-CAN transfer card. Auxiliary light source system, ultrasonic ranging module, differential GPS receiving module, and speed measurement block are connected to the industrial control computer through the RS485 bus. The robot is powered by the lithium battery, and each robot realizes data sharing through the wireless communication module.

The substation inspection robot carries a visible-light camera and an infrared thermograph which is used to detect invaders and equipments' fault point, respectively. The host PC plays a supervisory role, such as reading the status from each subsystem, recognizing the equipments from the thermal image, planning the inspection path, and displaying the 3D map. The on-board PC controls the behaviors of the robots, such as movement, obstacle avoidance, and positioning. The associated multirobot collaborative system adopts a hybrid layout, as shown in Figure 2. The host PC is responsible for computing, planning, and scheduling tasks. Information could be shared between the mobile robots over a wireless network.

2.2. Task Classification. We introduce three important inspection tasks here, which will be realized in Section 4 based on the proposed control methods designed in Section 3.

2.2.1. Single-Point Inspection. There are some important areas or fault-prone areas that need to be monitored in large substations of smart microgrids. For these areas, the inspection robot sometimes needs to carry out a singlepoint inspection. In this situation, the multirobot system assigns the task to a single robot according to the location of the inspection point, the location of each robot, and its ability value and then converts the problem into the path planning problem of the single robot in the known environment.

2.2.2. Multipoint Inspection. In real applications, there are generally more than one important or fault-prone areas that need to be inspected simultaneously. In this situation, the inspection task can be assigned to several robots according to the position of the task points; then, the reasonable paths are designed for each robot to complete the inspection task.
2.2.3. All-Area Inspection. If the entire equipment needs to be inspected, then the robot should move in the whole substation. In this situation, the basic process of multirobot inspection in a known environment includes the following: (a) grouping of multimobile robots in pairs; (b) design the opposite heuristic algorithm for each group of robots; (c) start the inspection, and when it falls into the dead zone, choose the IBENN method to jump out; and, (d) determine whether the entire area has been inspected.

In practical applications, no matter what kind of inspection task, the multimobile robot system needs to decompose and allocate tasks first; then, each robot separately formulates a reasonable inspection path. The commonly used path planning methods mainly include artificial potential field method, $A$-star algorithm, and fuzzy logic, which, however, have certain defects for different practical problems [28]. In order to accomplish various kinds of inspection tasks, the widely used BENN method will be introduced in the next part, and the IBENN algorithm will be proposed in Section 3.

\subsection{Biological Excitation Neural Network (BENN) Method.} In the bioexcitation neural network method, the motion space of a mobile robot can be represented by a topological state space composed of a neural network, and the activity value of neurons represents environmental information. The activity values of all neurons are generally initialized to 0 , while the changes in the activity values of each neuron are described by the following shunting equation $[29,30]$ :

$$
\frac{\mathrm{d} x_{i}}{\mathrm{~d} t}=-A x_{i}+\left(B-x_{i}\right)\left(\left[I_{i}\right]^{+}+\sum_{j=1}^{k} w_{i j}\left[x_{j}\right]^{+}\right)-\left(D+x_{i}\right)\left[I_{i}\right]^{-},
$$

where $x_{i}$ is the neural activity of the $i$ th neural, parameters $A$, $B$, and $D$ are nonnegative constants representing the passive decay rate, the upper and lower bounds of the neural activity, respectively, $k$ is the number of neurons in the neighborhood, $w_{i j}$ and $x_{j}$ represent the connection weight and activity of neurons in the neighborhood, respectively, $E$ is a normal number much larger than $B$, and $I_{i}$ is the $i$ th external input with the following form:

$$
I_{i}= \begin{cases}E, & \text { target; } \\ -E, & \text { obstacle; } \\ 0, & \text { other. }\end{cases}
$$

Excitation signal $S_{i}^{e}=\left[I_{i}\right]^{+}+\sum_{j=1}^{k} w_{i j}\left[x_{j}\right]^{+}$, and input inhibition $S_{i}^{i}=\left[I_{i}\right]^{-}$. The nonlinear threshold function $[a]^{+}$ is defined as $[a]^{+}=\max (a, 0)$ and $[a]^{-}=\max (-a, 0)$. Let $d_{i j}$ is the Euclidean distance between neurons, $w_{i j}=f\left(d_{i j}\right)$, and $f(a)=\left(u_{0} / a\right), u_{0}$ is a positive constant. The robot starts from the starting point to the next position $q_{n}$ determined by the following formula [31, 32]:

$$
q_{n} \Leftarrow x_{q_{n}}=\max \left(x_{j}, j=1,2,3, \ldots, k\right) .
$$

This method ensures that the positive neuronal activity value can spread outwards and affect the whole state space, 


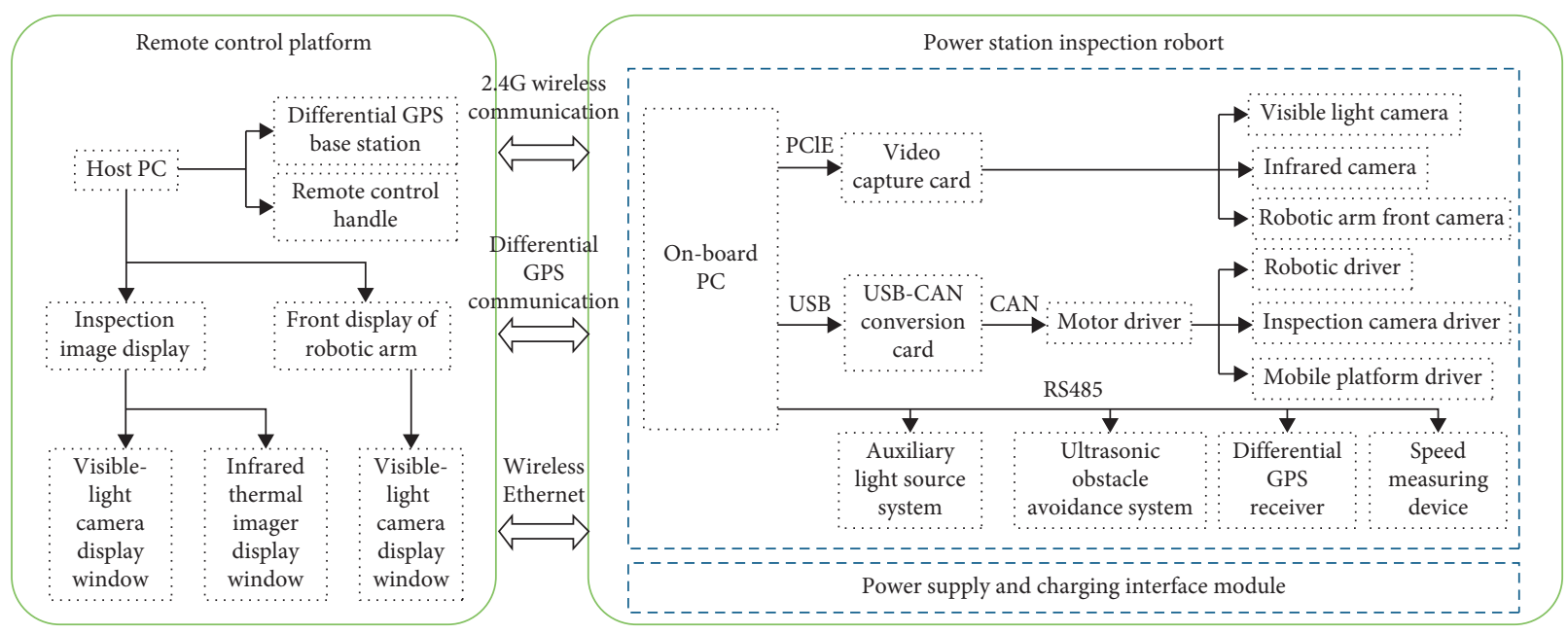

Figure 1: Single robot system architecture in power stations of smart microgrids.

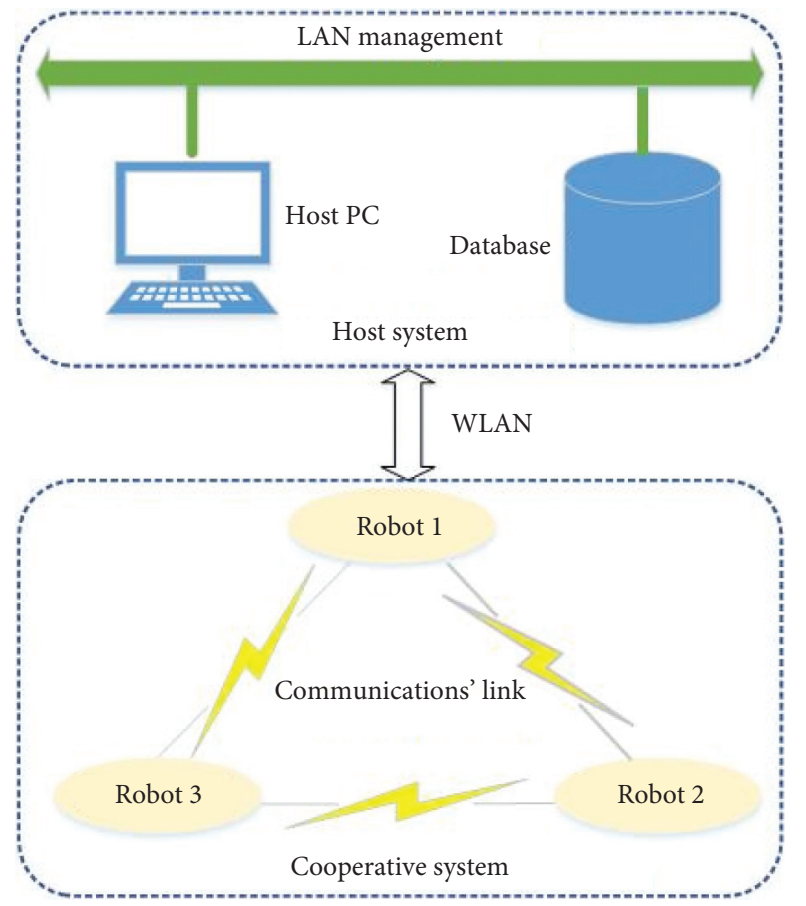

FIGURE 2: Multirobot system architecture in power stations of smart microgrids.

while the negative neuronal activity value can only act locally. The neuronal activity values of the target point and the obstacle are, respectively, at the crest and trough of the wave, as shown in Figure 3, so the target point can attract the robot to move towards it, while the obstacle can only repel the robot locally. After the robot reaches the next location from the present location, the next location becomes a new present location unless the present point is the target point. If the found next location is the same as the present location, the robot stays there without any movement.

Although this method can solve the problem of robot path planning, it still has some problems such as nonoptimal path, wrong path judgment, and easy to fall into the dead

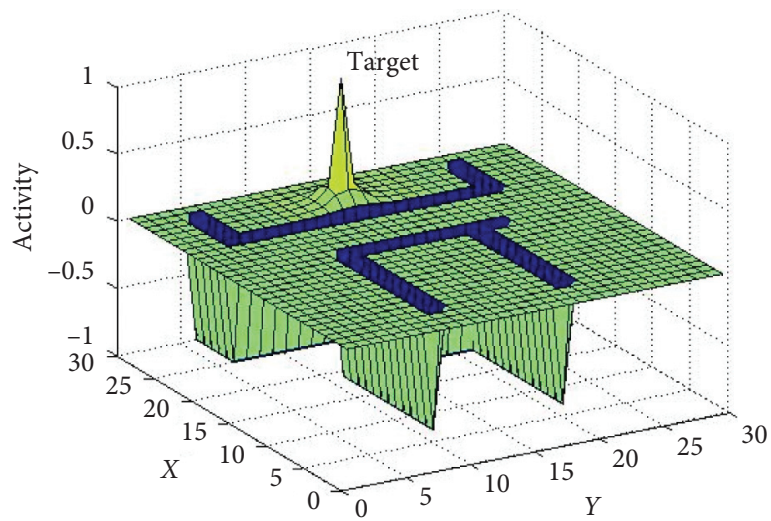

FIGURE 3: Biological excitation neuronal active field of the BENN method.

zone. To end this, an improved bioexcitation neural network algorithm is designed next, which will be applied for the multirobot to complete various types of smart grid inspection tasks.

\section{Improved Biological Excitation Neural Network (IBENN) Method}

When calculating the neuron activity value near the boundary, the traditional BENN method regards the boundary as an obstacle, making it relatively low relative to the surrounding nonboundary neuron activity value. As a result, the best paths are often overlooked, as shown in Figure 4. As seen, it is better to choose the path along the left boundary in Figure 4(a), and the optimal path is between the two obstacles in Figure 4(b). Moreover, the original method only considers the activity value of surrounding neurons when choosing the next path point, which ignores the robot's steering in the form process. However, robot steering requires deceleration, steering, and acceleration, which greatly increase the power consumption and travel time of the robot. 


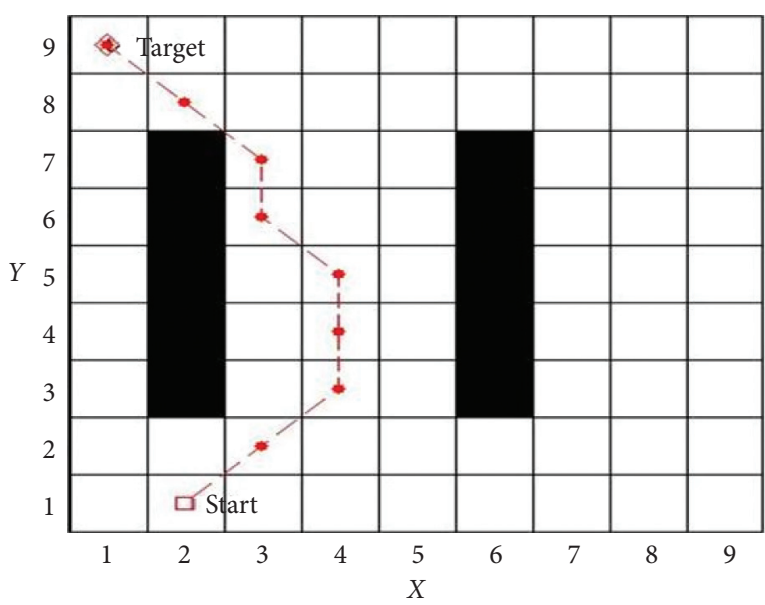

(a)

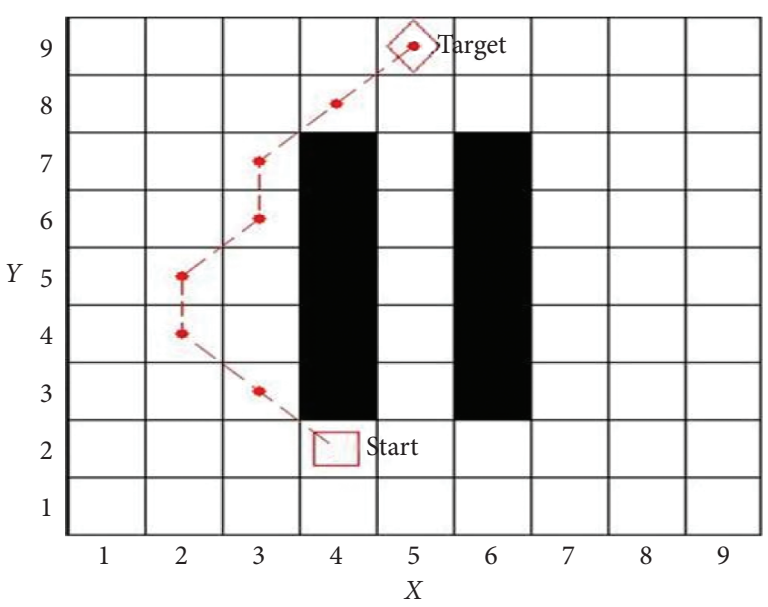

(b)

Figure 4: Unreasonable path planning under traditional methods. (a) Path along the boundary. (b) Obstacle intermediate path.

In order to reduce the power consumption of the robot and to smooth the path of the robot, the following path decision method is adopted:

$$
q_{n} \Leftarrow x_{q_{n}}=\max \left(x_{j}+c y_{j}, j=1,2,3, \ldots, k\right),
$$

where $x_{i}$ is the active value at the current position, $c$ is a positive constant, $y_{j}=1-\Delta \theta_{j} \pi$ is a monotonically increasing function of the moving direction difference between the current to the next robot, and $\Delta \theta_{j}$ is the absolute angle at which the robot moves from the current position to the next position, e.g., $\Delta \theta_{j}=0$ (or $\Delta \theta_{j}=\pi$ ) when the robot moves in a straight line (or turns around). Denote the coordinates of the current position $q_{c}=\left(x_{q_{c}}, y_{q_{c}}\right)$ and the previous position $q_{p}=\left(x_{q_{p}}, y_{q_{p}}\right)$, then

$$
\begin{aligned}
\Delta \theta_{j}= & \left|\theta_{j}-\theta_{c}\right|=\arctan \left(y_{q_{j}}-y_{q_{c}}, x_{q_{j}}-x_{q_{c}}\right) \\
& -\arctan \left(y_{q_{c}}-y_{q_{p}}, x_{q_{c}}-x_{q_{p}}\right) .
\end{aligned}
$$

The path decision method takes into account the factors of steering and selection of appropriate $c$, which represents the weight of steering in the path decision, only then a smooth path can be planned. In order to overcome the drawbacks of ignoring the boundary optimal path as well as unreasonable path selection, we propose the following improvement strategies:

Strategy (i). Map extension, i.e., extend the activity range of robot virtual neurons

Strategy (ii). Activity increasing, i.e., increase the activity of neurons near the obstacle and boundary

Strategy (iii). Node deletion, i.e., delete the redundant nodes that do not hinder the passage of robots

The boundary neurons of the original map are extended outward of one circle, respectively. These areas are assumed to be virtual reachable (not treated as obstacles) with the positive activity value and can also spread activity around during the initialization process, but are ignored when choosing the actual path. The main purpose of Strategies (i) and (ii) is to improve the activity value of the original map boundary neurons, which can be considered as a boundary protection for these neurons. In addition, if the robot can bypass the next adjacent node from a certain node without colliding with an obstacle in a path, the deletion of this node can effectively shorten the total length of the path, thus Strategy (iii) can reduce the robot's power consumption and improve work efficiency. To realize these improvements, the following Assumption and Definition are given.

Assumption 1. Neurons outside the boundaries of the original map are virtual nonobstacles, which also have the function of spreading activity values, and their activity values are the average activity values of nonobstacle points in the neighborhood.

Definition 1. If a robot is on a nine-square grid map-centered, the top and bottom, left and right, front left and back right, and front right and back left are, respectively, considered as four sets of symmetrical coordinates. If any pair of symmetrical coordinates are obstacles, the robot is said to be between the obstacles.

If the robot is between the obstacles, the excitation input of the neuron can be calculated by

$$
S_{i}^{e}=\left[I_{i}\right]^{+}+\frac{n \sum_{j=1}^{k} w_{i j}\left[x_{j}\right]^{+}}{n_{\text {pos }}} .
$$

When the neuron is not located between the obstacles, the excitation input of the neuron can be calculated by

$$
S_{i}^{e}=\left[I_{i}\right]^{+}+\frac{\left(n-n_{\text {neg }}\right) \sum_{j=1}^{k} w_{i j}\left[x_{j}\right]^{+}}{n_{\text {pos }}},
$$

where $n, n_{\text {pos }}$, and $n_{\text {neg }}$ represent the numbers of neighborhood neurons, nonobstacles, and obstacles, respectively. Let $S_{i}^{i}=\left[I_{i}\right]^{-}$, then system (1) can be rewritten as 


$$
\frac{\mathrm{d} x_{i}}{\mathrm{~d} t}=-A x_{i}+\left(B-x_{i}\right) S_{i}^{e}-\left(D+x_{i}\right) S_{i}^{i} .
$$

Hence, Strategies (i) and (ii) are used to increase the activity value of neurons near the boundaries or obstacles. In practical application, one of these two strategies can be selected and used together with Strategy (iii) to achieve better path planning.

Figure 5 shows the comparison experiment using Strategies (i) and (iii) with model parameters $A=10$, $B=D=1, E=100, k=8$, and $c=1$. The relevant data comparison is shown in Table 1. It can be seen from Figure 5 and Table 1 that the path length has been reduced from 10.07 to 8.41 , and the times of turns have been changed from 4 to 1 . Thus, the proposed strategies have obvious improvement effects.

In order to verify the superiority of Strategies (ii) and (iii), the simulation environment is set as a complex maze, and model parameters are also set as $A=10, B=D=1$, $E=100, k=8$, and $c=1$. The initial position of the robot is $(6,3)$, and the target position is $(1,30)$. The traditional BENN method, $A$-star algorithm, and the proposed IBENN method are compared, as shown in Figures 6 and 7. As seen, the proposed IBENN method cannot only solve the problem of path misjudgment but also reduce the path transition, smooth the path, and improve the path quality. Three index comparisons of path length, turn times, and total rotation angle are concluded in Table 2. It can be seen that, compared with the original BENN method, the path length, path turns, and total rotation angle of the proposed IBENN method are reduced by $6.12 \%, 41.2 \%$, and $64.3 \%$, respectively. Compared with the $A$-star algorithm, the number of turns and the total rotation angle can be reduced by $16.7 \%$ and $16.8 \%$, respectively, under the condition that the path length is unchanged. It concludes that the improved IBENN method shows better performance in terms of path quality and path smoothness than the other methods.

\section{Multirobot Cooperative Inspection (MRCI) System}

In this section, we will implement the proposed IBENN method to the MRCI system and complete the previously established three inspection tasks.

4.1. Single-Point Inspection. Assume that there are $n$ isomorphic inspection robots with different initial positions and $m$ areas that need to be monitored in a substation of smart microgrids, and one of the areas needs to be continuously monitored at a certain moment. In this situation, the system needs to schedule a robot to complete the task following the principle of minimum cost or maximum benefit. We establish the following mathematical model:

$$
\begin{aligned}
f_{i}(L, D) & =\min \left(\varepsilon L_{i j}+\eta D_{i j}, j=1,2,3, \ldots, g_{i}\right), \\
T_{i} \Leftarrow \cos t_{i} & =\min \left(f_{i}, i=1,2,3, \ldots, n\right),
\end{aligned}
$$

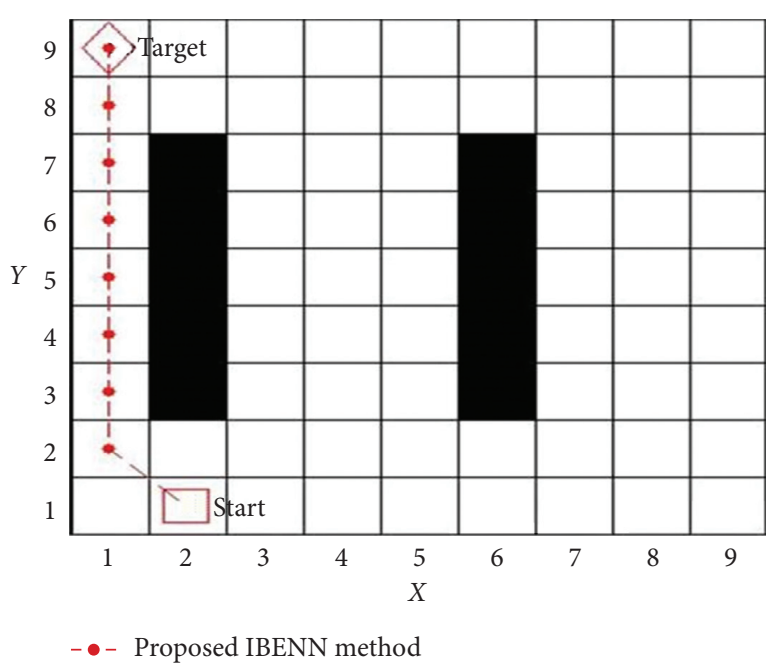

FIgURE 5: Single-robot path generated by the proposed IBENN method with Strategies (i) and (iii) in the situation of single-point inspection.

TABLE 1: Comparison results of BENN and IBENN methods.

\begin{tabular}{lcc}
\hline Evaluation index & BENN & IBENN with strategies (i) and (iii) \\
\hline Path length & 10.07 & 8.41 \\
Turn times & 4 & 1 \\
\hline
\end{tabular}

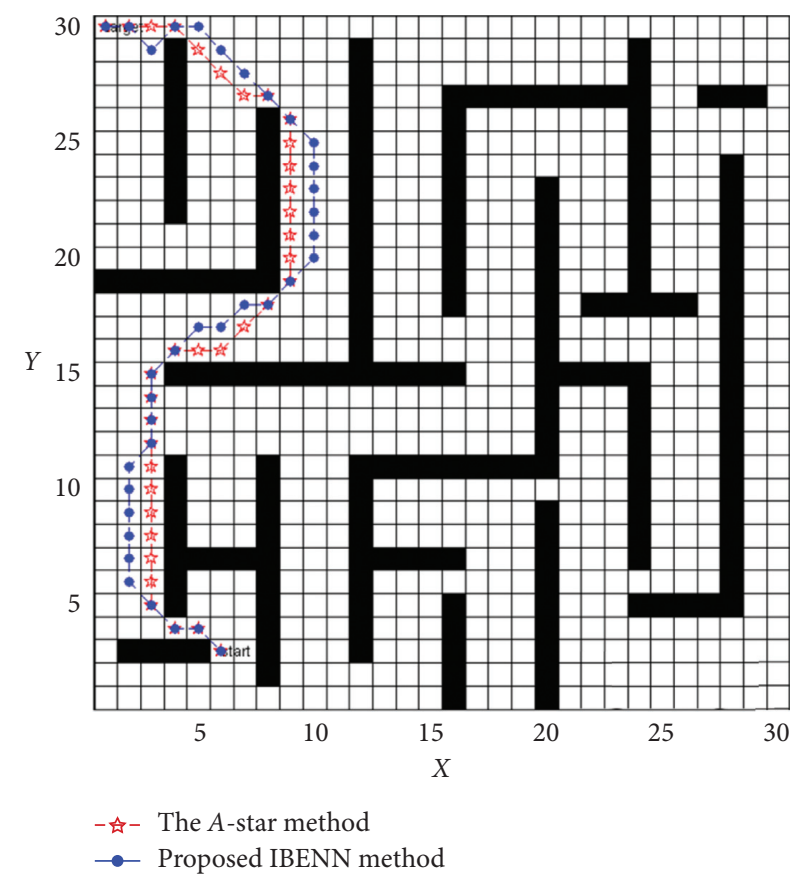

FIGURE 6: Single-robot path generated by the traditional BENN method and $A$-star algorithm in the situation of single-point inspection.

where $L_{i j}$ and $D_{i j}$ represent the length and the turning number of the $j$ th path for the $i$ th robot, respectively, $\varepsilon$ and $\eta$ represent the associated weights of $L_{i j}$ and $D_{i j}$, respectively, 


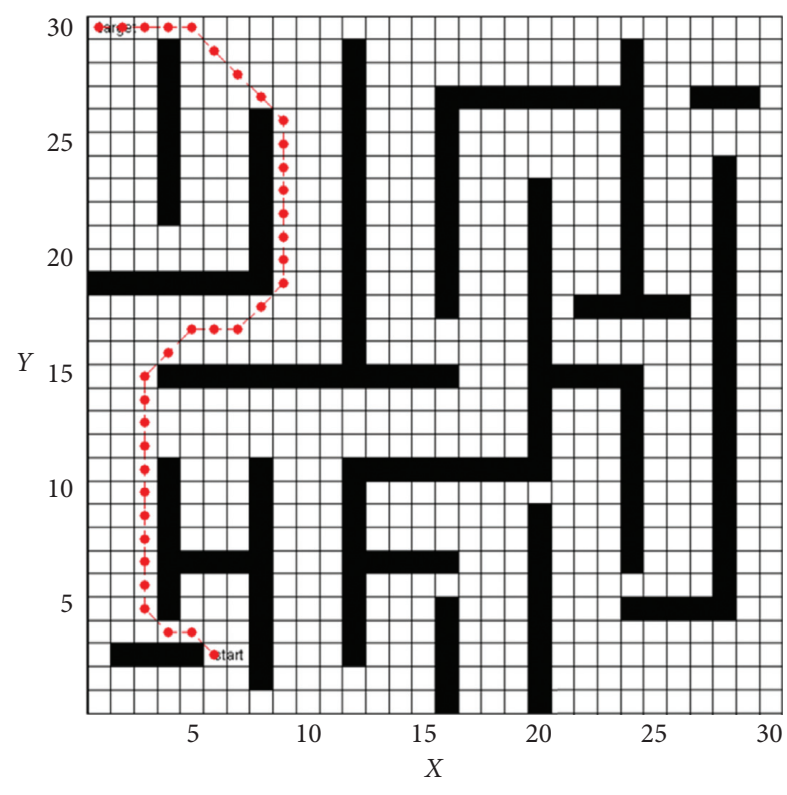

- - Proposed IBENN method

FIGURE 7: Single-robot path generated by the proposed IBENN method with Strategies (ii) and (iii) in the situation of single-point inspection.

TABle 2: Comparison of the three methods.

\begin{tabular}{lccc}
\hline Index & BENN & $\begin{array}{c}A- \\
\text { star }\end{array}$ & $\begin{array}{c}\text { IBENN with strategies (ii) and } \\
\text { (iii) }\end{array}$ \\
\hline $\begin{array}{l}\text { Path length } \\
\text { Turn times }\end{array}$ & 40.63 & 38.14 & 38.14 \\
$\begin{array}{l}\text { Rotation } \\
\text { angle }\end{array}$ & 21.99 & 9.43 & 10 \\
\hline
\end{tabular}

all robots' weights are the same by default in the path decision, $g_{i}$ represents the number of paths available for the $i$ th robot to complete the task $T$, and $f_{i}$ can be considered as the minimum cost of robot $i$ to achieve task $T$. The objective here is to find the robot $i$ and the optimal path $g_{i}$.

To solve this problem, the proposed IBENN method is used to calculate the minimum cost of each robot to complete the task and then compare the optimal cost of each robot. It is necessary that the robot with the lowest cost obtains the task and completes the task according to the optimal path planned. For example, in the $30 \times 30$ map shown in Figure 8, gray areas represent parallel devices and black ones represent obstacles in the site, and there are four robots, Robot 1, Robot $_{2}$, Robot $_{3}$, and Robot ${ }_{4}$, with initial positions, $R_{1}(1,1), R_{2}(30,1), R_{3}(1,30)$, and $R_{4}(30,30)$, respectively. When inspection tasks are needed near equipment $R_{\text {target }}(14,15)$ (i.e., area no. 9), according to the IBENN method, the cost of each robot to complete the task is calculated, as shown in Table 3, and then, each robot offers a quotation according to the above method with $f_{1}=14$, $f_{2}=14.14, f_{3}=12.06$, and $f_{4}=15.33$, which can be calculated according to (9) when the parameter is set as $\varepsilon=\eta=0.5$. According to the calculation, we can choose

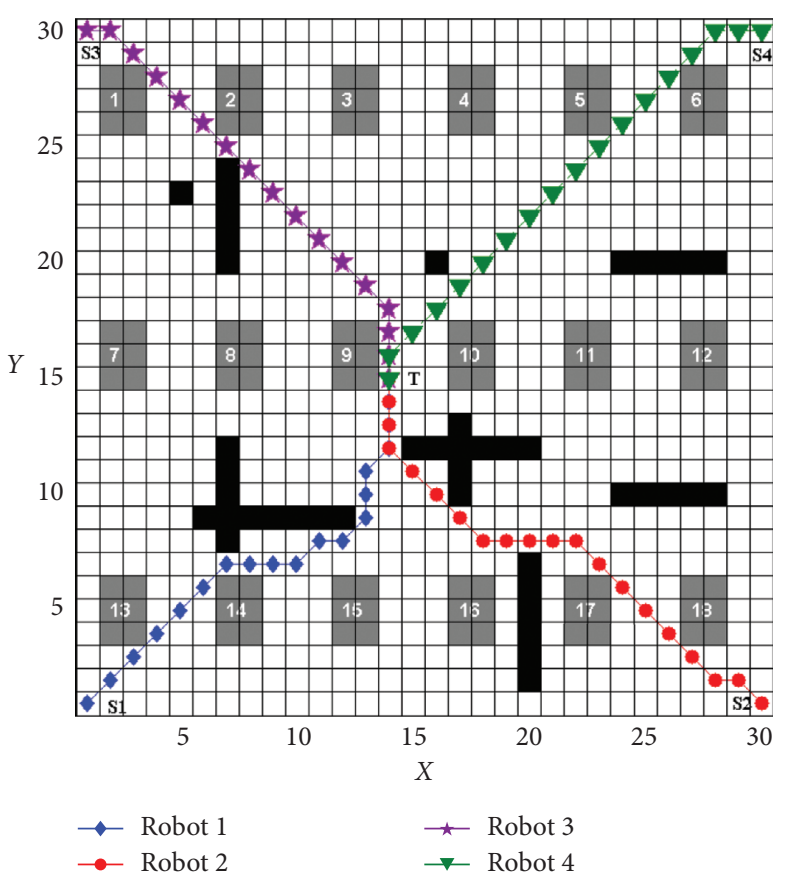

FIGURE 8: Four-robot path generated by the proposed IBENNbased MRCI system in the situation of single-point inspection.

TABle 3: Comparison of the four-robot path planning.

\begin{tabular}{lcccc}
\hline Index & Robot $_{1}$ & Robot $_{2}$ & Robot $_{3}$ & Robot $_{4}$ \\
\hline Path length & 21.73 & 23.56 & 20.97 & 22.80 \\
Turn times & 18 & 19 & 16 & 17 \\
Rotation angle & 6.28 & 4.71 & 3.14 & 7.85 \\
\hline
\end{tabular}

Robot $_{3}$ as the best scheme to execute the task, and other robots determine their priority as a backup according to the cost.

4.2. Multipoint Inspection. In the substation working area, there is occasionally a need to inspect multiple areas, and the advantages of the multirobot cooperation system can be well reflected in this situation. The cost equation of the system can be described as

$$
\begin{aligned}
F & =\min \cos t=\min \sum_{i=1}^{n} f_{i}\left(L_{i}, D_{i}\right) \\
& =\min \sum_{i}^{n}\left(\min \left(\varepsilon L_{i j}+\eta D_{i j}, j=1,2,3, \ldots, g_{i}\right)\right),
\end{aligned}
$$

where $g_{i}$ represents the total number of paths that robot $i$ can choose to reach the target point. Assume that there are $n$ robots with $m$ areas that need to be inspected $(n>1$ and $m>1)$ simultaneously, and the collaborative work of robots needs to be determined according to the relationship between the areas $m$ and the robots $n$. 
Case 1. $n \geq m$ : assume that a robot can only complete one task, and it can ensure that each uninspected area can be assigned to a robot to perform tasks at the same time. The allocation method is the same as the task allocation method of the single-point robot mentioned above, i.e., each robot puts forward an optimal quotation for each task, and the master robot determines the optimal decision-making scheme based on the quotation of each robot combined with the corresponding bidding algorithm and assigns subtasks to each robot according to the scheme. Then, the problem is first transformed into an assignment problem, which can be solved by the classical Hungarian algorithm [33]. For example, let $n=6$ and $m=4$, their positions are shown in Table 4, and the minimum cost of each robot at each task point is solved according to the above method. According to the above allocation method, the following allocation scheme can be obtained: $A_{1} \longrightarrow R_{1}, A_{2} \longrightarrow R_{2}$, $A_{3} \longrightarrow R_{3}$, and $A_{4} \longrightarrow R_{5}$, and the total cost $F=9.94+9.69+6.81+5.71=32.15$.

Case 2. $n<m$ : it means that a robot needs to complete multiple tasks, and there can also be a situation where one or more robots are idle. For multiple robots at different initial positions, the host computer needs to plan a reasonable path so that all inspection areas can be visited by the robots and the total cost is minimal. We have designed a genetic algorithm to solve this problem. The genetic algorithm adopts the following single-chain coding form, as shown in Fig-

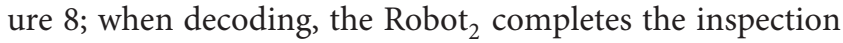
tasks of Areas 1 and 3 in sequence, and similarly, Robot ${ }_{1}$ and

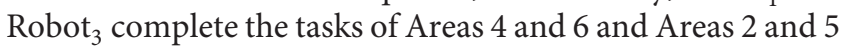
in sequence. This coding method has the advantage of strong readability; however, it is easy to produce infeasible solutions during crossover and mutation. Therefore, different repair algorithms are used to repair the infeasible solutions.

Crossover. Use Partial-Mapped Crossover (PMX) operator when generating a new gene chain. If it is not a feasible solution, then fix it. The specific repair method is to find the repetitive genes in gene chain A that cause infeasible solutions (there may be multiple groups), one of which must be in the cross-replacement gene sequence, then find the gene that maps to the gene in gene chain $B$, and use it to replace the repetitive genes in chain A that are not in the cross-replacement sequence.

Mutation. Randomly select two genes in the gene chain, and determine whether one of their positions is at the end of the gene chain. If not, exchange their positions in the gene chain.

Selection. The tournament selection method is used, that is, a certain number of individuals are removed from the population each time; then, the best one is selected to enter the offspring population. Repeat this operation until the new population size reaches the original population size.

For example, in a $30 \times 30$ map, the initial positions of the Robots $1-3$ are $R_{1}^{\text {start }}(1,10), \quad R_{2}^{\text {start }}(15,30), \quad$ and $R_{3}^{\text {start }}(30,10)$, and the positions of the 6 inspection points are
TABLE 4: Comparison of the four-robot path planning.

\begin{tabular}{lcccc}
\hline Position & Area $_{1}(9,16)$ & Area $_{2}(16,16)$ & Area $_{3}(22,7)$ & Area $_{4}(22,25)$ \\
\hline $\mathrm{R}_{1}(1,1)$ & 9.94 & 14.92 & 14.88 & 18.01 \\
$\mathrm{R}_{2}(15,1)$ & 10.31 & 9.69 & 7.88 & 16.88 \\
$\mathrm{R}_{3}(30,1)$ & 15.96 & 14.42 & 6.81 & 15.23 \\
$\mathrm{R}_{4}(1,30)$ & 11.01 & 11.58 & 19.87 & 12.71 \\
$\mathrm{R}_{5}(15,30)$ & 10.60 & 9.56 & 16.09 & 5.71 \\
$\mathrm{R}_{6}(30,30)$ & 25.18 & 14.41 & 15.51 & 11.32 \\
\hline
\end{tabular}

$A_{1}(11,27), A_{2}(29,28), A_{3}(3,18), A_{4}(16,15), A_{5}(13,3)$, and $A_{6}(26,6)$. The initial azimuth of the robot is $(0,-\pi / 2,-\pi)$. Using the proposed IBENN method above, the optimal path and the minimum cost between each point can be calculated. The genetic algorithm can draw the following distribution plan:

$$
R_{1} \longrightarrow A_{3} \longrightarrow A_{1} \longrightarrow A_{4} \longrightarrow A_{5} ; R_{2} \longrightarrow A_{2} ; R_{3} \longrightarrow A_{6}
$$

The total cost is 45.28 , and the multirobot multipoint inspection path is shown in Figure 9, where the red star indicates the inspection point and the blue star indicates the initial position of each robot.

4.3. All-Area Inspection. Since the substation of smart microgrids is exposed to the outdoors for a long time, it is easy to break into foreign objects or other dangerous goods. It is necessary to conduct regular inspections of the entire power station of smart microgrids for all-area coverage. However, performing this task by a single robot may take a long time and is inefficient. Therefore, a multirobot collaborative whole-area inspection program is designed.

Divide every two robots into a group, and design a heuristic algorithm for each robot. The priority of the heuristic algorithm of the robots in each group is opposite, which ensures that each group of robots can start inspections from different directions. When the robot enters the dead zone position, according to the proposed IBENN method above, the optimal path from the dead zone position to the uninspected area can be planned. The robot moves to this area and continues to inspect according to the original heuristic algorithm. For example, in a $30 \times 30$ map, there are parallel devices and various obstacle points, as shown in Figure 10. Two robots in the area are used to coordinate to complete the all-area inspection task. The steering priority of the heuristic algorithm of Robot $_{1}: W-S-S W-N W-N-$ $E-S E-N E$ and Robot $2: E-N-N E-S E-S-W-N W$ $-S W$, and the initial positions are $(1,1)$ and $(30,1)$, respectively.

Simulation experiments were carried out in the obstacle-free and obstacle environments, and the results are shown in Figures 11 and 12 and Table 5, where the red and blue represent Robots 1 and 2, respectively, pink dots express repeated path points, dots mean the final position of the robot when the task is completed, and stars represent the initial position. It can be seen from Table 5 that, in a simple map, when there are no other obstacles in the inspection area and only parallel substation equipment, 


\begin{tabular}{|l|l|l|l|l|l|l|l|l|}
\hline $\mathrm{A}_{1}$ & $\mathrm{~A}_{3}$ & $\mathrm{R}_{2}$ & $\mathrm{~A}_{4}$ & $\mathrm{~A}_{6}$ & $\mathrm{R}_{1}$ & $\mathrm{~A}_{2}$ & $\mathrm{~A}_{5}$ & $\mathrm{R}_{3}$ \\
\hline
\end{tabular}

Figure 9: Gene chain coding of the genetic algorithm.

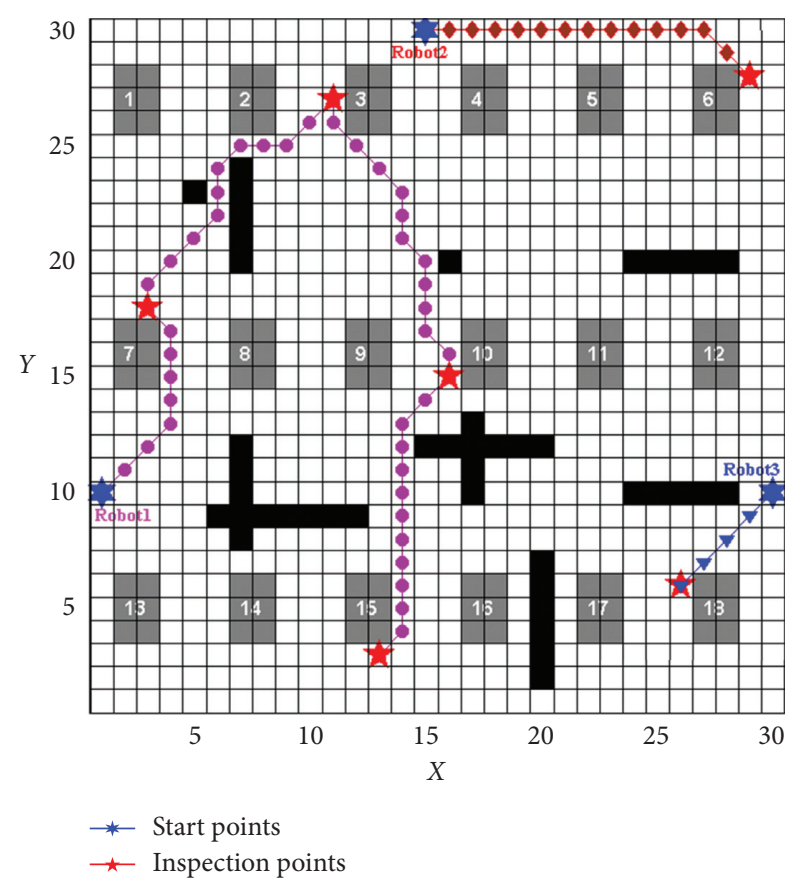

FIgURE 10: Three-robot path generated by the proposed IBENN based MRCI system in the situation of six-point inspection.

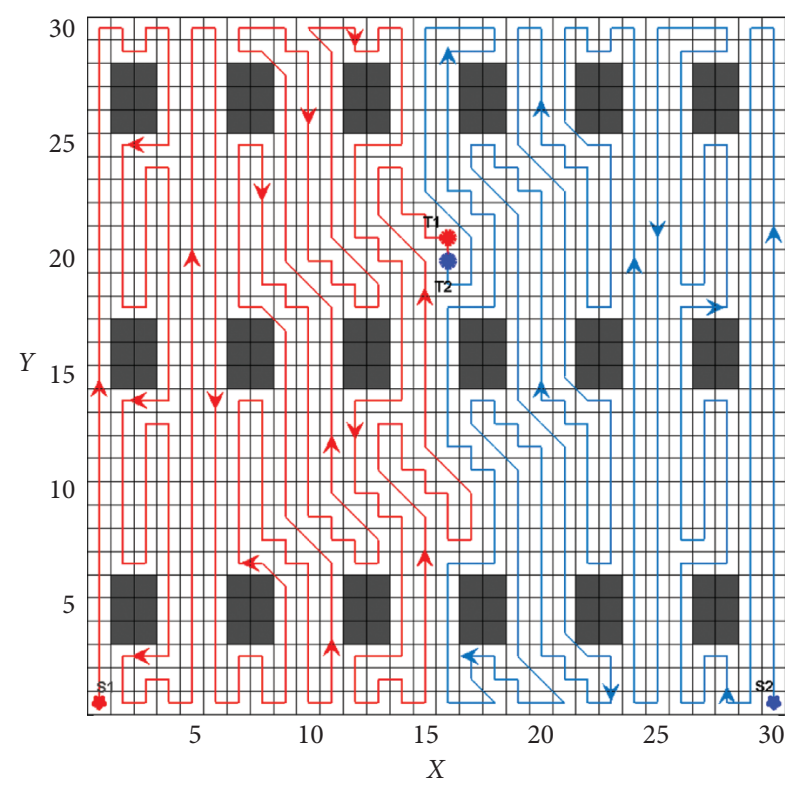

FIgURE 11: Two-robot path generated by the proposed IBENNbased MRCI system in the situation of all-area inspection without obstacle.

this method can achieve 100\% coverage without overlapping areas. When there are various obstacles in the inspection area, this method can also achieve 100\% full-

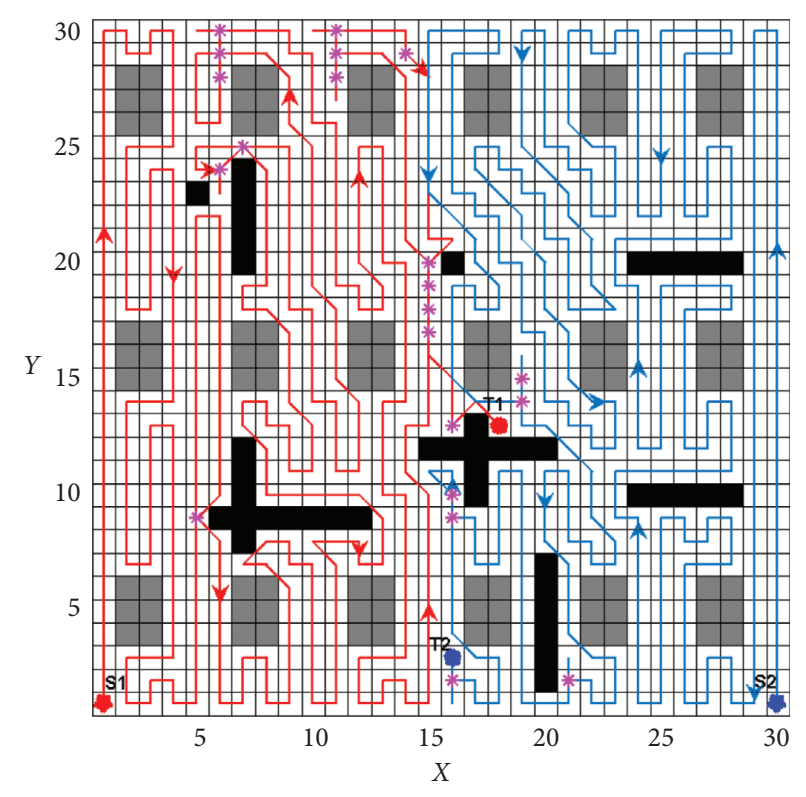

FIGURE 12: Two-robot path generated by the proposed IBENNbased MRCI system in the situation of all-area inspection complex obstacle.

TABLE 5: Results in obstacle-free and obstacle environments.

\begin{tabular}{lcc}
\hline $\begin{array}{l}\text { Evaluation } \\
\text { index }\end{array}$ & $\begin{array}{c}\text { Obstacle-free } \\
\text { environment (\%) }\end{array}$ & $\begin{array}{c}\text { Obstacle } \\
\text { environment (\%) }\end{array}$ \\
\hline Coverage rate & 100 & 100 \\
Repetition rate & 0 & 2.3 \\
\hline
\end{tabular}

coverage inspection, but there is a repetition rate of $2.3 \%$. Hence, compared with other inspection methods, the proposed IBENN method does not need to set a template operator and has strong versatility. Multirobot collaboration can complete the entire area and full-coverage inspection task.

To further verify the effectiveness of the proposed control schemes, we carry out the simulation experiment with Coppelia Sim Software in the space of $30 \mathrm{~m} \times 30 \mathrm{~m}$. The two inspection robots are in the initial position as $t=0$, as shown in Figure 13(a), in which the gray cube and dark blue cube represent the equipment area and the obstacle area, respectively. The robot is supposed to inspect at a speed of $1 \mathrm{~m} / \mathrm{s}$ disregarding the inspection time. Using the above inspection strategy, the experimental results are shown in Figure 13. As seen, the left robot and right robot fall into dead zones, respectively, as $t=138$ or $t=365$ in Figures 13(a) and 13(e) and $t=357$ or $t=306$ in Figures 13(c) and 13(d). However, in these cases, the inspection machine can jump out of the dead zone by calling the IBENN algorithm, respectively. Furthermore, both robots fall into the dead zone state when $t=395$, as shown in Figure 13(f). Since there is no undetected area in this situation, it follows that the inspection task has been completed. Therefore, the experiment verifies the feasibility of the cooperation scheme. 


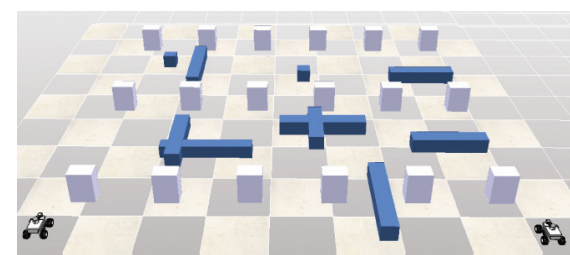

(a)

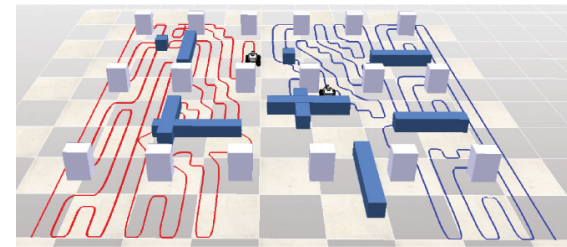

(c)

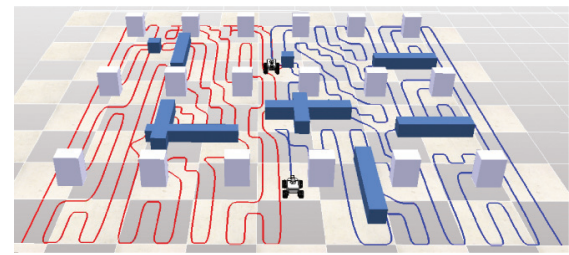

(e)

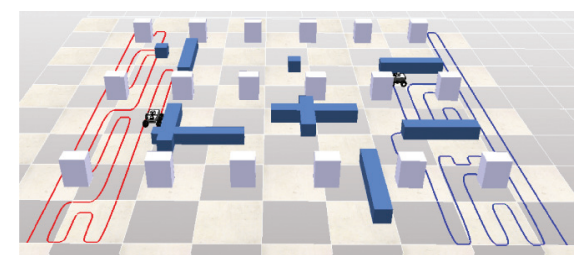

(b)

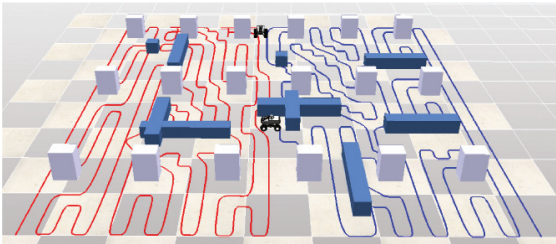

(d)

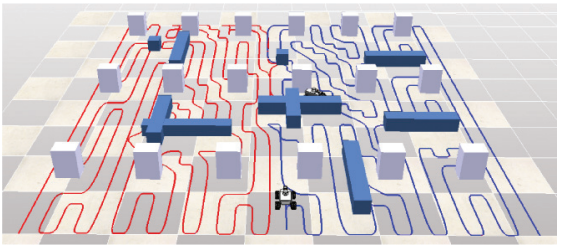

(f)

Figure 13: Sequence diagram of cooperative operation of two inspection robots. (a) $\mathrm{T}=0$. (b) $\mathrm{T}=138$. (c) $\mathrm{T}=306$. (d) $\mathrm{T}=357$. (e) $\mathrm{T}=365$. (f) $\mathrm{T}=395$.

\section{Conclusion}

In this paper, a multimobile robot collaborative operating system for power stations of the smart microgrids is designed, and an improved IBENN method is proposed. Compared with the traditional BENN method and $A$-star algorithm, the improved IBENN algorithm has an obvious optimization effect in path length and turning times. Based on this method, the multirobot cooperative operation strategy is designed to realize the three classic tasks of singlepoint inspection, multipoint inspection, and full-coverage inspection in the substation of smart microgrids, which verifies the effectiveness of the main results.

\section{Data Availability}

All of the data used to support the findings of this study are included within this article.

\section{Conflicts of Interest}

The authors declare that they have no conflicts of interest.

\section{Acknowledgments}

This work was supported by the National Natural Science Foundation of China under Grant nos. 61733004 and 61773158.

\section{References}

[1] A. Peyman and F. Keynia, "Lifetime efficiency index model for optimal maintenance of power substation equipment based on cuckoo optimisation algorithm," IET Generation Transmission \& Distribution, vol. 11, no. 11, pp. 2787-2795, 2017.

[2] I. Ullah, Y. Fang, and R. Khan, "Predictive maintenance of power substation equipment by infrared thermography using a machine-learning approach," Energies, vol. 10, no. 12, pp. 1-13, 1987.

[3] X. Lu, J. Lai, X. Yu, Y. Wang, and J. M. Guerrero, "Distributed coordination of islanded microgrid clusters using a two-layer intermittent communication network," IEEE Transactions on Industrial Informatics, vol. 14, no. 9, pp. 3956-3969, 2018.

[4] X. Lu, J. Lai, X. Yu, Y. Wang, and J. M. Guerrero, “A novel secondary power management strategy for multiple AC microgrids with cluster-oriented two-layer cooperative framework," IEEE Transactions on Industrial Informatics, vol. 17, no. 2, pp. 1483-1495, 2021.

[5] H. Takahashi, Development of Patrolling Robot for Substation, pp. 10-19, Japan IERE Council,Special Document R8903, Japan, 1989.

[6] J. Beaudry and S. Poirier, Véhicule téléopéré pour inspection visuelle etthermographique dans les postes de ransformation, 2012.

[7] J. K. C. Pinto, M. Masuda, L. C. Magrini, J. A. Jardini, and M. V. Garbelloti, "Mobile robot for hot spot monitoring in electric power substation," in Proceedings of the IEEE/PES Transmission and Distribution Conference and Exposition, pp. 1-5, Chicago, Illinois, April 2008.

[8] N. Pouliot, P.-L. Richard, and S. Montambault, "LineScout technology opens the way to robotic inspection and maintenance of high-voltage power lines," IEEE Power and Energy Technology Systems Journal, vol. 2, no. 1, pp. 1-11, 2015.

[9] A. Cantieri, M. Ferraz, and G. Szekir, "Cooperative UAVUGV autonomous power pylon inspection: an investigation of cooperative outdoor vehicle positioning architecture," Sensors, vol. 20, no. 6384, pp. 1-22, 2020. 
[10] Y. Guang and P. Yutian, "Intelligent inspection of marine disasters based on UAV intelligent vision," Journal of Coastal Research, vol. 93, pp. 410-416, 2019.

[11] S. Jordan, J. Moore, and S. Hovet, "State-of-the-art technologies for UAV inspections," IET Radar, Sonar \& Navigation, vol. 12, no. 2, pp. 151-164, 2018.

[12] J. Katranik, F. Pernus, and B. Likar, "A survey of mobile robots for distribution power line inspection," IEEE Transactions on Power Delivery, vol. 25, no. 1, pp. 485-493, 2010.

[13] J. Yu and S. M. LaValle, "Optimal multirobot path planning on graphs: complete algorithms and effective heuristics," IEEE Transactions on Robotics, vol. 32, no. 5, pp. 1163-1177, 2016.

[14] K. Jose and D. K. Pratihar, "Task allocation and collision-free path planning of centralized multi-robots system for industrial plant inspection using heuristic methods," Robotics and Autonomous Systems, vol. 80, pp. 34-42, 2016.

[15] B. Pradhan, A. Nandi, N. B. Hui, and D. S. Roy, "A novel hybrid neural network-based multirobot path planning with motion coordination," IEEE Transactions on Vehicular Technology, vol. 69, no. 2, pp. 1319-1327, 2020.

[16] C. Luo, S. X. Yang, and X. Li, "Neural-dynamics-driven complete area coverage navigation through cooperation of multiple mobile robots," IEEE Transactions on Industrial Electronics, vol. 64, no. 1, pp. 750-760, 2017.

[17] B. Woosley and P. Dasgupta, "Integrated real-time task and motion planning for multiple robots under path and communication uncertainties," Robotica, vol. 36, no. 3, pp. 353-373, 2018.

[18] B. Woosley, P. Dasgupta, J. G. Rogers, and J. Twigg, "Multirobot information driven path planning under communication constraints," Autonomous Robots, vol. 44, no. 5, pp. 721-737, 2020.

[19] F. Imeson and S. L. Smith, "An SMT-based approach to motion planning for multiple robots with complex constraints," IEEE Transactions on Robotics, vol. 35, no. 3, pp. 669-684, 2019.

[20] A. Ayari and S. Bouamama, "A new multiple robot path planning algorithm: dynamic distributed particle swarm optimization," Robotics and Biomimetics, vol. 4, no. 1, p. 8, 2017.

[21] B. Banerjee and CE. Davis, "Multiagent path finding with persistence conflicts," IEEE Transactions on Computational Intelligence and AI in Games, vol. 9, no. 4, pp. 402-409, 2016.

[22] S. Thabit and A. Mohades, "Multi-robot path planning based on multi-objective particle swarm optimization," IEEE Access, vol. 7, pp. 2138-2147, 2018.

[23] R. Siegwart, "IR nourbakhsh, and D scaramuzza. "Introduction to autonomous mobile robots," Industrial Robot, vol. 2, no. 6, pp. 645-649, 2004.

[24] K. Jiang, Z. Sun, Y. Liu, and J. Sui, "Development and Application of the Rail-type Inspection Robot used in Substation Rooms," MATEC Web of Conferences, vol. 139, no. 5, Article ID 00210, 2017.

[25] H. B. Xu, J. H. Liu, and X. Jia, "Substation inspection system based on RFID technology," Advanced Materials Research, vol. 734-737, pp. 2846-2850, 2013.

[26] S. Z. Wang, D. Han, and Q. Dong, "The application of RFID in substation inspection system," Applied Mechanics and Materials, vol. 295-298, pp. 2433-2436, 2013.

[27] J. Zhu, Y. Sun, and D. Sun, "Design of a laser navigation system for the inspection robot used in substation," Proceedings of the Spie, vol. 322, 2017.

[28] C.-J. Huang, Y.-W. Wang, H.-M. Chen et al., "Application of cellular automata and type-2 fuzzy logic to dynamic vehicle path planning," Applied Soft Computing, vol. 19, pp. 333-342, 2014.

[29] S. X. Yang and M. Meng, "An efficient neural network approach to dynamic robot motion planning," Neural Networks, vol. 13, no. 2, pp. 143-148, 2000.

[30] S. X. Yang and M. Q.-H. Meng, "Real-time collision-free motion planning of a mobile robot using a Neural Dynamicsbased approach," IEEE Transactions on Neural Networks, vol. 14, no. 6, pp. 1541-1552, 2003.

[31] S. Yang and C. Luo, A Neural Network Approach to Complete Coverage Path Planning, pp. 718-724, Systems Man and Cybernetics, 2004.

[32] C. Luo and S. X. Yang, "A bioinspired neural network for realtime concurrent map building and complete coverage robot navigation in unknown environments," IEEE Transactions on Neural Networks, vol. 19, no. 7, pp. 1279-1298, 2008.

[33] S. Chopra, G. Notarstefano, M. Rice, and M. Egerstedt, "A distributed version of the Hungarian method for multirobot assignment," IEEE Transactions on Robotics, vol. 33, no. 4, pp. 932-947, 2017. 